\title{
The Association Among Structural Quality, Operational Competence And Emergency Outpatient Visits: A Longitudinal Analysis Of US Community Hospitals
}

Aber Elsaleiby, The University of Illinois at Urbana Champaign \& Washington University in St. Louis, USA

\begin{abstract}
The aim of the current study is to enhance our understanding on how hospital's structural quality and operational competence can influence the emergency outpatient visits. We use a large database acquired from the American Hospital Association. The database has state-wide data on several health care quality parameters of interest over an extended period of 17 years starting from 1994 till 2010 for all community hospitals in the US. (4,926 community hospitals). Structural quality is captured by a measure of bed availability and usage. Operational competence is measured by the expense per capita. Finally, data on emergency outpatient visits is our outcome measure. We found that there is a significant negative association between structural quality and emergency outpatient visits. Similarly, there is a significant negative association between hospital operational performance and emergency outpatient visits. It is confirmed in this study that at high level of operational performance, increasing the structural quality will be associated with lower emergency outpatient visits. The interaction effect between structural quality and operational performance on emergency outpatient visits is also significant. As such, structural quality of hospitals can reduce emergency outpatient visits. Hospitals endeavor to improve its operational performance also reduces emergency outpatient visits. Joint emphasis on improving bed utilization and improving operational performance can further decrease emergency outpatient visits.
\end{abstract}

Keywords: Bed Utilization; Structural Quality; Expense Per Capita; Emergency Outpatient Visits

\section{INTRODUCTION AND THEORETICAL BACKGROUND}

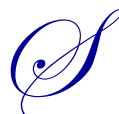

ervice is a corner stone of economic activities in any society and is considered one of the most significant economic sectors in the United States. (Li, Benton, \& Leong 2002). Despite the importance of healthcare in the service sector, it has been struggling with several quality and cost related problems. The Institute of Medicine (I.O.M.) reported how the American healthcare sector had been experiencing quality and cost related problems to the degree that about $10 \%$ of US community hospitals closed in the 1990's because of the poor performance (IOM 2001). The American Hospital Association (AHA) stated that community hospitals cost in the US increased dramatically to $\$ 1002.50$ billion in 2012 with a mean expense per capita of $\$ 3675$ compared to $\$ 540.80$ billion in total expense, and a mean expense per capita of $\$ 8925$ in 1994 (AHA, 2014).

As a matter of fact, quality in the healthcare context has long been identified as difficult to define and accordingly hard to measure (Scott \& Flood, 1984; Donabedian, 1988; Theokary \& Ren, 2011). This is mainly due to the particular nature of healthcare as a complex service industry that involves different highly interacting work units (Iezzoni, Davis, Soukup \& O’Day 2002; Tucker, Nembhard \& Edmondson, 2007). One of the earliest and most comprehensive definitions of healthcare quality was provided in 1980 by Donabedian who defined healthcare quality as a care expected to "maximize an inclusive measure of patient welfare, after one has taken account of the balance of expected gains and losses that attend the process of care in all its parts." Donabedian showed that quality of healthcare delivery (i.e. outcome quality) can be affected by the hospital environment and resources as well as the technical methods and guidelines (Donabedian, 1980). In 2009, Porter further emphasized Donabedian's concept by pinpointing the 
importance of maximizing the value delivered to patients while cutting down cost associated with the unnecessary use of the resource. According to the value based care approach, the joint focus on what to deliver (i.e. the clinical aspect of delivering care), how to deliver it, and at what cost are three mail pillars to value based care (Porter, 2009).

Research has indicated that higher number of emergency visits is linked to longer waiting times, higher mortality rates and higher cost (Williams, 1996; Bernstein, Aronsky, Duseja \& Epstein 2008; Armour, 2015). Emergency room (ER) problems have attracted the attention of researchers who continue to pinpoint the need for a methodologically sound study of emergency department visits, flow, and cost (Williams, 1996; Bamezai, Melnick \& Nawathe 2005; Bamezai \& Melnick, 2006). Nationally, ER outpatient visits exceeds 125 million (www.cdc.gov) and it is still going up in the third year of the Affordable Care Act, refuting the law's supporters who had expected a decline in traffic as more people gained access to doctors and other health-care providers (Armour, 2015). Patients may return visits to the ER if their initial medical condition deteriorates, the initial care provided to them in the ER is inadequate, or if they are rashly discharged. This, of course, can lead to increase in the ER visits. Increased number of ER visits is linked to "crowding" that contribute to delays, medical errors and adverse events (Bernstein et al. 2008). Researchers have found that better management of emergency outpatient visits can help hospital cut down unnecessary cost. Hence, identifying hospital related factors that contribute to better management of emergency outpatient visits is very valuable from the hospital standpoint.

In the current study, we try to explore the relationship among structural quality, expense per capita, and the emergency outpatient visits. More specifically, structural quality is measured using surrogates, specifically, bed availability and utilization, two of the components identified by Donabedian (1980). Nair, Malhotra \& Ahire (2011) have identified expense per capita as a reflective measure of hospital operational competence capturing the hospital's endeavor and commitment to process and administrative quality. Kellermann \& Jones (2013) hypothesize that ER outpatient visits is a measure of output quality, with low rate indicating that the community hospital is doing a satisfactory job of maintaining the community's health which results in lower ER visits and vice-versa. Hence, outcome quality is approximated by ER outpatient visits. We use data from 5000 state-wide community hospitals over an extended period of seventeen years.

The paper is organized as follows section 2 addresses the research methodology showing the data used and the model developed in this paper. Results of the regression analysis are presented in Section 3. Discussion of the results and the managerial implication are shown in Section 4.

\section{METHODS}

\subsection{Data Description}

Data are collected from American Hospital Association (AHA) over a seventeen year period from the year 1994 till 2010. The data includes different quality parameters that we operationalized in our model from about 5000 US Registered Community Hospitals categorized by state. Community hospitals account for $86.9 \%$ of US hospitals, and their expenses account for $91 \%$ of total expenses of US hospitals (AHA, 2013). Community hospitals include all nonfederal, short-term general, and other specialized hospitals that include obstetrics and gynecology; eye, ear, nose, and throat; rehabilitation; orthopedic; and other individually described specialty services. They also include academic medical centers or other teaching hospitals if they are nonfederal short-term hospitals. Excluded are hospitals not accessible by the general public, such as prison hospitals or college infirmaries (AHA, 2013).

A snippet of the data used for all fifty states over the stated period is presented in Table 1. Data is normalized into per ' 000 of population. We anticipate that with 17 years of data spread over 50 states, some variables under study might slightly change from one year to another for a given state and hence proper grouping and agglomeration of data becomes vital. In order to deal with this issue, we initially divide the longitudinal data into two almost equal sized time periods (1994-2001 and 2002-2010) in an effort to check whether the relationships are stationary. 
Table 1. Average values of health care quality indicators over the two periods (1994-2001, 2002-2010) by state using raw data (Selected states are only shown)

\begin{tabular}{l|c|c|c|c|c|c|c|c|c}
\hline \multirow{2}{*}{ State } & \multicolumn{2}{|c|}{ Number of Beds } & \multicolumn{2}{c|}{ Utilization } & \multicolumn{2}{c|}{ Expense Per Capita } & \multicolumn{2}{c}{$\begin{array}{c}\text { Emergency } \\
\text { Outpatient Visits }\end{array}$} \\
\cline { 2 - 10 } & $\mathbf{1 9 9 4 - 2 0 0 1}$ & $\mathbf{2 0 0 2 - 2 0 1 0}$ & $\mathbf{1 9 9 4 - 2 0 0 1}$ & $\mathbf{2 0 0 2 - 2 0 1 0}$ & $\mathbf{1 9 9 4 - 2 0 0 1}$ & $\mathbf{2 0 0 2 - 2 0 1 0}$ & $\mathbf{1 9 9 4 - 2 0 0 1}$ & $\mathbf{2 0 0 2 - 2 0 1 0}$ \\
\hline Alabama & 4 & 3 & 0.588 & 0.618 & 1160 & 1607 & 466 & 477 \\
\hline Alaska & 2 & 2 & 0.605 & 0.578 & 997 & 1981 & 377 & 478 \\
\hline Arizona & 2 & 2 & 0.610 & 0.677 & 862 & 1399 & 299 & 324 \\
\hline West Virginia & 4 & 4 & 0.602 & 0.617 & 1376 & 2144 & 604 & 640 \\
\hline Wisconsin & 3 & 3 & 0.588 & 0.627 & 1145 & 1995 & 340 & 359 \\
\hline Wyoming & 4 & 4 & 0.527 & 0.547 & 930 & 1558 & 403 & 430 \\
\hline Max. & $\mathbf{7}$ & $\mathbf{6}$ & $\mathbf{0 . 7 7 8}$ & $\mathbf{0 . 8 1 6}$ & $\mathbf{3 3 5 9}$ & $\mathbf{5 0 7 2}$ & $\mathbf{6 6 1}$ & $\mathbf{6 8 5}$ \\
\hline Min. & $\mathbf{2}$ & $\mathbf{2}$ & $\mathbf{0 . 5 2 7}$ & $\mathbf{0 . 5 3 1}$ & $\mathbf{7 9 7}$ & $\mathbf{1 3 0 1}$ & $\mathbf{2 4 1}$ & $\mathbf{2 7 0}$ \\
\hline Average & $\mathbf{3}$ & $\mathbf{3}$ & $\mathbf{0 . 6 2 5}$ & $\mathbf{0 . 6 4 9}$ & $\mathbf{1 1 7 8}$ & $\mathbf{1 9 2 6}$ & $\mathbf{3 9 2}$ & $\mathbf{4 1 7}$ \\
\hline
\end{tabular}

Further, we based our analysis on the following three main measures for each quality indicator for each state: (1) average value over the time period under study, (2) ranks of average values to enable us to capture subtle differences among states more effectively, and (3) the compound growth rate of the raw data as given in the data base over specified period of time. In order to capture the whole picture more accurately over that extended period of time, we calculate the rate of change over the targeted time span using a compound growth (or decay) rate of data over the two time intervals (1994-2001 and 2002-2010).

\subsection{Data Transformation}

Publically available yearly data from AHA for all 50 states and DC on several measures on a per ' 000 of population were used in this study. Raw data were not sufficiently discriminatory in their scope and accordingly could not reveal fine differences among states. In order to study differences in the health indices over such a long period of time, we divide the data into two sets, the first set covers the period 1994-2001 while the second set covers 2002-2010. Further, we average the data within each set and rank the averaged data in either ascending or descending order depending on the index under study. For consistency, we generally assign the lowest rank to the best quality indicator. More specifically, we posit that a state that has higher number of beds will be better in terms of bed availability and therefore we rank the different states in decreasing order with respect to the average number of beds per ' 000 . We assume that a hospital with the highest average number of beds per ' 000 will have at least one component of structural quality and will be regarded as the best with respect to this quality indicator and accordingly will be ranked as number one state. With respect to the utilization of beds, it is assumed that the hospital that has a low bed utilization will be able to admit more patients with minimum waiting time which in turn will be reflected on patient satisfaction regarding the hospital performance. Hence, states are arranged in increasing order based on their average calculated values of beds utilization using the logic that the hospital with the lowest bed utilization (more patient satisfaction) will have the highest level of structural quality based on this component and again quality rank number one will be assigned to the state with the lowest bed utilization. Then, we add the two ranks to obtain a composite ranking for structural quality. The lowest combined rank represents the highest structural quality.

Regarding the expense per capita indicator which includes payroll, non-payroll, bad debt and all non-operating expenses, we hypothesize that a hospital with high expense per capita will be better off in terms of process quality because higher expense per capita imply more financial compensation for staff, more financial resources to be spent on training that will facilitate adhering to established practice guidelines and will lead to higher process quality (Donabedian, 1980; Tucker et al., 2007). A state which scores highest expanse per capita will be ranked number one to reflect the best potential for process quality and operational performance.

The number of emergency outpatient visits is used in the current study to reflect the outcome quality following Kellermann, Hsia, Yeh \& Morganti (2013) who found that a well-structured emergency department will be providing a lifesaving care on a continuous basis and will be associated with better outcome quality. Accordingly, we hypothesize that a state with low emergency outpatient visits will be regarded as having better outcome quality. Table 2 exhibits the summery for the ranking of the different variables incorporated into the model. 
Table 2. Index for the quality parameters ranks

\begin{tabular}{l|c}
\hline \multicolumn{1}{c|}{ Health indicator } & Best quality perceived when \\
No. of beds & Highest rank \\
\hline Utilization (non-availability) & Lowest rank \\
\hline Expense per capita & Highest rank \\
\hline Number of emergency outpatient visits & Highest rank \\
\hline
\end{tabular}

\section{ANALYZING THE EMPIRICAL RELATIONSHIPS}

\section{What Relationship Exists Among the Healthcare Quality Parameters?}

Our study of hospital quality may be seen as being grounded on industrial quality literature and the relevant quality standards. Specifically, they are analogous to the principle used in ISO-9000 according to which an acceptable level of outcome quality of a certain product will be expected given that the input of good quality raw materials and having used good quality processes, that are standardized and documented.

We use the number of beds per ' 000 of the population and utilization of beds per ' 000 of the population as surrogate measures for structural quality. As mentioned earlier, we overcome the lack of sufficient discriminatory power in the raw data using the rank of each healthcare quality indicator. More specifically, we ranked states in decreasing order with respect to their average number of beds per ' 000 population arguing that the hospital with the highest average number of beds per ' 000 population will have at least one component of structural quality which will be considered the best with regarding this quality measure. In addition, we rank the utilization in increasing order of their averages based on the logic that the hospital with the lowest utilization can admit patients with minimum waiting and hence will have the highest level of structural quality concerning this component. The two ranks are then added to obtain a composite ranking for structural quality.

As previously mentioned, expense per capita includes payroll, non-payroll, bad-debt (most likely to be cost of uncompensated services to indigents and uninsured) and non-operating expenses. A hospital with higher expenses has more healthcare skillful employees who are better trained. We postulate that such hospitals with higher expense per capita would have a higher level of process quality. Again, data on this measure is ranked in descending order of average expenses such that the smaller rank, the better process quality is expected to be.

Ideally, literature identifies outcome quality it in terms of changes to health status attributable to care received (i.e. Donabedian, 1980; Chandrasekaran, Senot \& Boyer 2012). Measures of outcome quality include rates of morbidity, mortality, infection, and re-hospitalization (Donabedian, 1980; Scott \& Flood, 1984; Iezzoni et al. 2002). Recently, Kellermann et al. (2013) reported that up-to-date emergency departments are able to provide "around-the-clock lifesaving care" in individual emergencies and disasters in addition to the timely diagnostic workups, "after-hours acute care", and serve as the "safety net of the safety net" for millions of low-income and uninsured patients. As such, the number of emergency outpatient visits was used in the current study to reflect the outcome quality in the sense that lower number of emergency outpatient visits will be associated with lower morbidity, infection, complication and other rehospitalization drivers and accordingly better outcome quality (Donabedian, 1980; Baker, 2001; Iezzoni et al. 2002). A state with low emergency outpatient visits will be regarded as having better outcome quality. Therefore, we arranged the different states in ascending order with respect to the average number of emergency outpatient visits over the specified period. A state with the lowest number of emergency outpatient visits will be assigned rank number 1 to denote it as the state with the best outcome quality.

Based on the previous discussion we developed the model shown in Figure 1 which depicts the relationship among the healthcare quality dimensions namely structural quality, process quality and outcome quality. We hypothesize that:

H1: Structural quality is negatively related to the number of emergency outpatient visits.

H2: Hospitals' operational performance is negatively related to the emergency outpatient visits. 
H3: Hospitals' structural quality positively moderates the relationship between hospitals' operational performance and emergency outpatient visits. In other words, the interaction effect between structural quality and hospitals' operational performance is positively related to outcome quality.

Figure 1. The conceptual model

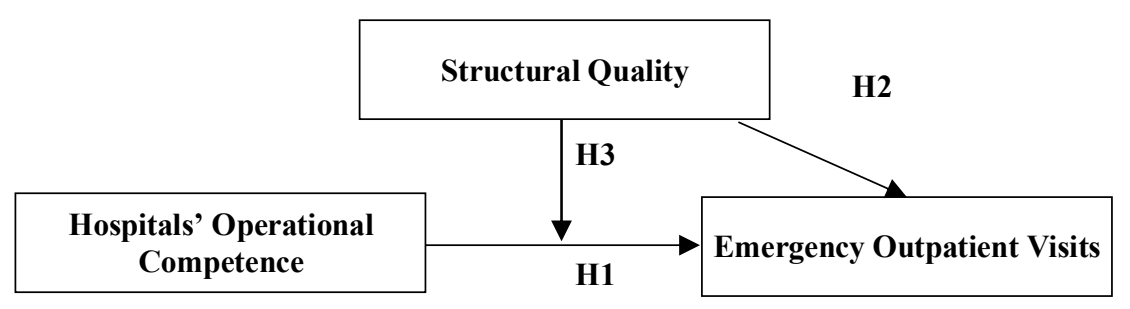

\section{RESULTS}

A paired t-test was performed on the two sets of data to test if each of the indicators under study differs over the two periods, namely 1994-2001 and 2002-2010. As shown in Table 3 the data for the two periods are significantly different, thus providing a justification for dividing the seventeen years data set into two approximately equal sized sets for our study.

Table 3. Paired sample t-test for two sets of data (1994-2001) and (2002-2010) using the raw data

\begin{tabular}{|c|c|c|c|c|c|c|}
\hline $\begin{array}{l}\text { Indicators tested over the two periods } \\
(1994-2001) \text { and }(2002-2010)\end{array}$ & Mean & $\begin{array}{l}\text { Std. } \\
\text { Deviation }\end{array}$ & $\begin{array}{l}\text { Std. Error } \\
\text { Mean }\end{array}$ & $\mathbf{t}$ & df & $\begin{array}{l}\text { Sig. } \\
\text { (2-tailed) }\end{array}$ \\
\hline Number of Beds & 0.373 & 0.488 & 0.0684 & 5.449 & 50 & \multirow{3}{*}{$\mathrm{P}<0.0001$} \\
\hline Emergency outpatient visits & 24.671 & 15.021 & 2.103 & -11.729 & 50 & \\
\hline Expense per Capita & 748.725 & 238.088 & 33.339 & -22.458 & 50 & \\
\hline
\end{tabular}

We initially test the effect of the number of beds on the rank of the emergency outpatient visit which is found to be not significant. This can be interpreted through the resource based view of the firm that states that availability of resources by itself cannot drive desired output without the complementarity with the different activities inside the firm. In a hospital setting, more bed availability may not lead to improved performance, but improved bed utilization may lead to improved performance. As such, we included the beds utilization in our model instead of the number of beds available. Our research model assumes a nonlinear relationship among our quality indicator. The research model is in the form:

$$
\hat{Z}=a * X_{1}^{b} * X_{2}^{c}
$$

taking the natural log for both sides will enable us to transform model (2) into a logarithmic model in the form:

$$
\log \hat{Z}=\log a+b^{*} \log X_{1}+c^{*} \log X_{2}
$$

We tested model (2) using multiple hierarchical regression analysis for the ranked data for the variables under study. In order to test hypothesis 3 , we included the interaction effect of both bed utilization and emergency outpatient visits. We conduct separate analysis for each time period. Output of the regression analysis is presented in Table 4 for both time period. 
Table 4. Regression results

\begin{tabular}{l|l}
\hline \multicolumn{1}{c}{ Data set (02-10) } & $-0.179^{* *}$ \\
\hline Structural Quality & $-0.266+$ \\
\hline Operational Performance & $-0.413+$ \\
\hline Structural Quality* Operational Performance & 0.307 \\
\hline R Square & 0.263 \\
\hline Adjusted R Square & \\
\hline Data set (94-01) & $-0.257^{* * *}$ \\
\hline Structural Quality & $-0.412^{* * *}$ \\
\hline Operational Performance & $-2.720^{* *}$ \\
\hline Structural Quality* Operational Performance & 0.219 \\
\hline R Square & 0.187 \\
\hline Adjusted R Square &
\end{tabular}

As seen from Table 4, there is a significant negative association between structural quality and emergency outpatient visits $(\beta=-0.179$ for $02-10$ data set, $p<0.05$ and $\beta=-0.257$ for $94-01$ data set, $p<0.001)$ for both time periods. Similarly, there is a significant negative association between hospital operational performance and emergency outpatient visits $(\beta=-0.266$ for $02-10$ data set, $p<0.10$ and $\beta=-0.412$ for $94-01$ data set, $p<0.001)$ for both time periods. at high level of operational performance, increasing the structural quality will be associated with lower emergency outpatient visits.

The interaction effect between structural quality and operational performance on emergency outpatient visits is also significant ( $\beta=-0.413$ for $02-10$ data set, $p<0.10$ and $\beta=-2.720$ for $94-01$ data set, $p<0.05$ ), thus providing support to hypothesis 3 .

\section{DISCUSSION AND MANAGERIAL IMPLICATIONS}

Our research combined insights from operations management literature, healthcare literature utilizing the data set from American Hospital Association (AHA). Our proposed research model and findings provide very important implications in healthcare setting. This study covers community hospitals which represent $86.9 \%$ of US hospitals thus provides generalizable results as to how structural quality and operational performance should be jointly emphasized to reduce the emergency outpatient visits. Structural quality embodies bed availability and utilization. The operational competence is measured through the dollar amount per capita that is spent on improving staff practices and enhancing the environment through which healthcare service is delivered.

Our findings suggest that managers and policy makers should focus on the proper utilization of their non-financial resources (i.e. bed) to guarantee the desired level of output. Within this context, it should be mentioned that past research found that financial resources are needed to promote healthcare quality in general, however, researchers and practitioners were always concerned about what activities a hospital should spend on, when, and by how much (Donabedian 1988). Accordingly, it is our contention that it is the responsibility of medical professionals, government agencies and advocacy organizations to work along well defined framework that comprises three main pillars: first, a hospital should have a technique through which it can identify the right activities that needed to be performed to achieve the maximum healthcare quality, second, the hospital should prioritize these activities based on criticality, ease of application, and anticipated level of output, and finally hospitals should determine the relevant cost associated with carrying out such activities. A healthcare decision maker should start their journey by performing the activity that will provide highest profit margin for each dollar spent towards enhancing the hospital quality.

\section{REFERENCES}

Armour, S. (2015). U.S. emergency-room visits keep climbing. Available at: http://www.wsj.com/articles/u-s-emergency-roomvisits-keep-climbing-1430712061

American Hospital Association (2013). AHA annual survey database. American Hospital Association, Chicago, IL.

American Hospital Association (2014). Trendwatch chartbook: Trends affecting hospitals and health systems. Available at http://www.aha.org/research/reports/tw/chartbook/2014/14chartbook.pdf

Baker, A. (2001). Crossing the quality chasm: A new health system for the 21st century. BMJ, 323(7322), 1192. 
Bamezai, A., Melnick, G., \& Nawathe, A. (2005). The cost of an emergency department visit and its relationship to emergency department volume. Annals of Emergency Medicine, 45(5), 483-490.

Bamezai, A., \& Melnick, G. (2006). Marginal cost of emergency department outpatient visits: An update using California data. Medical Care, 44(9), 835-841.

Bernstein, S. L., Aronsky, D., Duseja, R., Epstein, S., Handel, D., Hwang, U., ... \& Asplin, B. R. (2009). The effect of emergency department crowding on clinically oriented outcomes. Academic Emergency Medicine, 16(1), 1-10.

Chandrasekaran, A., Senot, C., \& Boyer, K. K. (2012). Process management impact on clinical and experiential quality: Managing tensions between safe and patient-centered healthcare. Manufacturing \& Service Operations Management, 14(4), 548-566.

Donabedian, A. (1980). Explorations in quality assessment and monitoring. Vol.1: The Definition of Quality and Approaches to its Assessment. Ann Arbor MI Health Administration Press.

Donabedian, A. (1988). The quality of care: How can it be assessed? Jama, 260(12), 1743-1748.

Institute of Medicine (IOM). (2001). Crossing the quality chasm: A new health system for the 21st Century. Institute of Medicine, Washington DC: National Academy Press.

Iezzoni, L., Davis, R. Soukup, J. \& O’Day, B. (2002). Satisfaction with quality and access to health care among people with disabling conditions. International Journal for Quality in Health Care, 14(5), 369.

Kellermann, A. L., \& Jones, S. S. (2013). What it will take to achieve the as-yet-unfulfilled promises of health information technology. Health Affairs, 32(1), 63-68.

Kellermann, A. L., Hsia, R. Y., Yeh, C., \& Morganti, K. G. (2013). Emergency care: Then, now, and next. Health Affairs, 32(12), 2069-2074.

Li, L. X., Benton, W. C., \& Leong, G. K. (2002). The impact of strategic operations management decisions on community hospital performance. Journal of Operations Management, 20(4), 389-408.

Nair, A., Malhotra, M. K., \& Ahire, S. L. (2011). Toward a theory of managing context in Six Sigma process-improvement projects: An action research investigation. Journal of Operations Management, 29(5), 529-548.

Porter, M. E. (2009). A strategy for health care reform - toward a value-based system. New England Journal of Medicine, 361(2), 109-112.

Scott, R., \& B. Flood. (1984). Costs and quality of hospital care: A review of the literature. Medical Care Review, 41(4), $213-61$.

Theokary, C., \& Justin Ren, Z. (2011). An empirical study of the relations between hospital volume, teaching status, and service quality. Production and Operations Management, 20 (3), 303-318.

Tucker, A. L., Nembhard, I. M., \& Edmondson, A. C. (2007). Implementing new practices: An empirical study of organizational learning in hospital intensive care units. Management Science, 53(6), 894-907.

Williams, R. M. (1996). The costs of visits to emergency departments. New England Journal of Medicine, 334(10), $642-646$.

The National Center for Health Statistics. http://www.cdc.gov/nchs/fastats/hospital.htm. Accessed: July 18, 2016 


\section{NOTES}

